American Journal of Agricultural and Biological Sciences 6 (1): 134-141, 2011

ISSN 1557-4989

(C) 2010 Science Publications

\title{
Screening of Pseudomonas sp. Isolated from Rhizosphere of Soybean Plant as Plant Growth Promoter and Biocontrol Agent
}

\author{
${ }^{1}$ Aris Tri Wahyudi, ${ }^{1}$ Rika Indri Astuti and ${ }^{2}$ Giyanto \\ ${ }^{1}$ Department of Biology, Faculty of Mathematics and Natural Science, \\ ${ }^{2}$ Department of Plant Protection, Faculty of Agriculture, \\ Bogor Agricultural University, Jl. Agatis-Kampus IPB Darmaga, Bogor 16680, Indonesia
}

\begin{abstract}
Problem statement: Pseudomonas spesies are one of the rihizobacterial group that have an important role in plant growth promoter and plant health. To prepare them as inoculants, they must have a range of characters as growth promoter such as Indole Acetic Acid (IAA) producers which can promote the growth of plants and solubilize phosphates. In addition, they must also have the various characters that act as biocontrol agents such as siderofor, chitinase and anti-fungal compound producers. Approach: Pseudomonas sp isolated from soybeans rhizospere and identified based on physiological reactions and 16S rRNA gene sequences. Various tests for the determination of the growth promoter were based on IAA production, phosphate solubilization and growth promoter of length of root and stems and number of lateral roots of soybean sprouts. Test of siderophore, chitinase, as well as anti anti-fungal compounds productions to inhibit the growth of Fusarium oxysporum, Rhizoctonia solani and Sclerotium rolfsii, were used as a biocontrol agent determination. Hypersensitivity test was used to screen for Pseudomonas sp classified as non-pathogenic rhizobacteria. Results: Fourteen isolates identified as a non-pathogenic Pseudomonas sp that produced IAA and Promoted enhancement of root length, shoot length, or number of lateral root. Among those 14 isolates, 8 isolates showed phosphate solubilizing activity, 12 isolates capable of producing siderophore and six isolates were observed to have chitinolytic activity. Only three isolates were able to inhibit the growth of Fusarium oxysporum in high level. While one and two isolates inhibited Sclerotium rolfsii and Rhizoctonia solani in high level, respectively. Conclusion: On the basis of excellent growth promoter and biocontrol activities, we recommended 5 isolates of Pseudomonas sp which were Crb-3, Crb-16, Crb-17, Crb-44 and Crb-94 as potential isolates of Pseudomonas sp that could be applied as inoculants of soybean plant.
\end{abstract}

Key words: Rhizobacteria, Pseudomonas sp, growth promoter, biocontrol, soybean, Indole Acetic Acid (IAA), Plant Growth Promoting Rhizobacteria (PGPR), Analysis Of Variance (ANOVA), Solubilizing Index (SI), Phosphate Solubilizing Bacteria (PSB), Induced Systemic Resistance (ISR), rRNA gene

\section{INTRODUCTION}

Bacteria that inhabit the rhizosphere may influence plant growth by contributing to a host plant's endogenous pool of bioactive compounds such as phytohormones, antibiotics, siderophores (Patten and Glick, 2002; Mubarikf et al., 2010). Those kind of bacterial group are well-known as Plant Growth Promoting Rhizobacteria (PGPR). PGPR can exhibit a variety of characteristics responsible for influencing plant growth (Ahmad et al., 2005). PGPR are considered to promote plant growth directly or indirectly. Indirect effects are related to production of metabolites, such as antibiotics, siderophores, or HCN, that decrease the growth of phytopathogens and other deleterious microorganisms. Direct effects are dependent on production of plant growth regulators or improvements in plant nutrients uptake (Bai et al., 2003).

Some genera of bacteria have been determined as PGPR including Bacillus, Pseudomonas, Azospirillum, Azotobacter, Bradyrhizobium, Rhizobium. An effective PGPR should have at least three characters of promoting plant activities which are root colonization

Corresponding Author: Aris Tri Wahyudi, Division of Microbiology, Department of Biology, Faculty of Mathematics and Natural Sciences, Bogor Agricultural University (IPB), Jl. Agatis-Kampus IPB Darmaga, Bogor 16680, Indonesia Tel/Fax: +62-251-8622833 
competency, phytostimulator and biocontrol agent against plant pathogens (Bloemberg and Lugtenberg, 2001). Pseudomonas sp. is one of the most important members of PGPRs showing all the three major group of PGPRs. Pseudomonas sp has been reviewed for the biofertilizer, phytostimulator and phytopathogen biocontrol activities. Direct plant growth activities of Pseudomonas sp include the production of Indole Acetic Acid (IAA) (Vasanthakumar and McManus, 2004) and siderophore (Dey et al., 2004), phosphate solubilization (Wu et al., 2005), ACC deaminase, root elongation, degradation of toxic compound (Bano and Musarrat, 2003) and as biological control agent for phytophatogens such as Aspergillus niger and A. flavus (Dey et al., 2004).

According to the remarkable PGPR characters of Pseudomonas sp, therefore in this study we isolated Pseudomonas sp. from the rhizosphere of soybean plant. The isolates were evaluated for phytostimulator and biocontrol capabilities in order to gain potential inoculants to enhance crop yields. The objectives of this study were to (i) isolate Pseudomonas sp from the soybean rhizosphere, (ii) screen the abilities and the possession of plant growth promoting attributes include the production of IAA hormone and siderophore, root elongation, phosphate solubilization, chitinase activity and the ability to inhibit pathogenic fungi Fusarium oxysporum, $R$. solani and Sclerotium rolfsii the causal agent of root rot disease in soybean. The $16 \mathrm{~S}$ rRNA analysis was also conducted in this study in order to determine species taxa of several isolates.

\section{MATERIALS AND METHODS}

Growth media and culture conditions: All isolated Pseudomonas spp. were maintained in King'S B medium and grown at room temperature. Whereas, all phyto-pathogenic fungi ( $R$. solani, $S$. rolfsii and $F$. oxysporum) were grown on PDA (potato dextrose agar) at room temperature for 3-5 days of incubation.

Isolation of Pseudomonas sp: Rhizosphere soil samples were collected from rhizosphere of soybean field in Plumbon, Cirebon, Indonesia. Rhizosphere soil samples were screened for Pseudomonas spp. using dilution method with King's B Agar as semi selective medium. Pseudomonas spp. isolates were estimated by morphological and physiological characteristics based on Bergeys' Manual of Systematic Bacteriology.

\section{Growth promoter assay:}

IAA Measurement: IAA was measured using the method described by Patten and Glick (2002) with slight modifocation. Pseudomonas spp. isolates were cultured in Kings'B broth supplemented with tryptophan $0.5 \mathrm{mM}$ for $48 \mathrm{~h}$. The measurement of IAA was done by spectrophotoscopy at $520 \mathrm{~nm}$ using Salkowsky reagent $\left(150 \mathrm{~mL} \mathrm{H} \mathrm{SO}_{4}, 7,5 \mathrm{~mL} \mathrm{FeCl}\right.$. $6 \mathrm{H}_{2} \mathrm{O} 0,5 \mathrm{M}$ and $250 \mathrm{~mL}$ aquadest). The detection of IAA was determined by the development of pink color. IAA concentration of each sample was estimated by compared to IAA st andard curve.

Germination seed assay: Seedling bioassay was conducted based on the method described by (Dey et al., 2004). For seedling bioassay, each Pseudomonas spp. isolate, was grown in King's B medium agar plates at room temperature for $24 \mathrm{~h}$. The inoculants for treating seeds were prepared by suspending cells from agar plates in nutrient broth in order to gain approximately $10^{10}$ cell per $\mathrm{mL}$. Nine surface-sterilized pre-germinated seeds (2-3 $\mathrm{mm}$ of radicula) per Petri dish with three replications for each treatment were prepared and subsequently drooped with $100 \mu \mathrm{L}$ suspension of bacterial cell. Germinating parameters were measured after 7 days of incubation including the length of the primary root, shoot and numbers of lateral roots. The data results were analyzed statistically with one-way Analysis of Variance (ANOVA) and further analyzed with Duncan Test $(\alpha=0.05)$ using SPSS 15 program.

Phosphate solubilizing assay: Solubilization of tricalcium phosphate was detected in Pikovskaya's Agar medium (Rao and Sinha, 1962). Each isolate of Pseudomonas spp. was streaked in Pikovskaya agar medium and incubated for 24-48 h. The development of clear zone around the colony indicated phosphate solubilizing activity. The solubilizing activity was expressed as Solubilizing Index (SI).

Hypersensitivity test: About $100 \mu \mathrm{L}$ of culture suspension of each Pseudomonas spp. isolates (24-48 h of incubation) was injected in to intercellular space between veins vessel of tobacco leaves. This treatment was conducted for three replications. As negative control, we injected sterilized aquadest and Escherichia coli in the other side of tobacco leaves, whereas Ralstonia solanacearum was also used as positive control. Positive hypersensitivity response was exhibited by dryness and brown necrosis at tissue's leaf after $24 \mathrm{~h}$ of incubation. Meanwhile, the development of yellow color in tissue's leaf indicated no hypersensitive response (Lelliott and Stead, 1987).

\section{Biocontrol assay:}

Siderophore production: Siderophore production was tested qualitatively using chrome azurol $\mathrm{S}$ medium 
(CAS-medium) (Husen, 2003). There are four important solutions that must be prepared in order to make CAS medium. Those four solutions were Fe-CAS solution, buffer solution, nutrition solution and cassamino acid solution. At $50^{\circ} \mathrm{C}$ after autoclaving, solutions 3 (nutrition) and 4 (cassamino acid) were added to the buffer solution. Indicator solution (solution-1) was added last with sufficient stirring to mix the ingredients. This mixture (Fe-CAS dye complex) yielded blue to dark green color. Each Pseudomonas spp. isolates were streaked on the surface of CAS medium and incubated for $48 \mathrm{~h}$. The production of siderophore was indicated by orange halos around the colonies.

Chitinase production: Chitinase production was investigated by used chitin medium (Cattelan et al., 1999). The Pseudomonas spp. isolates were prepared by cultivating on King's B medium for $24 \mathrm{~h}$ on shaking incubator. About $1 \mu \mathrm{L}$ suspension of Pseudomonas spp. was dropped in to surface of chitin agar medium. Chitinolytic activity was determined by the the development of clear zone around the Pseudomonas spp. colony. The chitinolytic index was measured by using formula: ( $\varnothing$ clear zone- $\varnothing$ colony) $\div \varnothing$ colony. ( : diameter).

Antagonism assay against plant root pathogenic fungi: Each Pseudomonas spp. isolates was assayed for antifungal activities against Fusarium oxysporum, Rhizoctonia solani and Sclerotium rolfsii using Potato Dextrose Agar (PDA) with method described by Dikin et al. (2006). Pseudomonas spp. isolates were streaked on PDA medium $3 \mathrm{~cm}$ in distance opposite to pathogenic fungi inoculated at the center of the medium. The barrier between Pseudomonas spp. isolate and pathogenic fungi indicated antagonist interaction between them. Antagonist activity was investigated for 4-7 days. The value of inhibition was measured using the formula as described by Ramesh et al. (2002):

$$
\text { InhibitionIndex }=\left[1-\left(\frac{\mathrm{A}}{\mathrm{B}}\right)\right] \times 100 \%
$$

Where:

$\mathrm{a}=$ Distance between fungi in the center of Petri dish to Pseudomonas spp. Isolate

$\mathrm{b}=$ Distance between fungi in center of Petri dish and blank area without Pseudomonas spp. isolate

Genetic analysis

16S-rRNA gene amplification: Isolation of Pseudomonas spp. genome was conducted with CTAB method (Wilson, 1994). Amplification of 16S rRNA gene was carried out with generic 16S rRNA primer described by Marchesi et al. (1998). Primer 63f (5'CAG GCC TAA CAC ATG CAA GTC-3') and 1387r (5'- GGG CGG WGT GTA CAA GGC-3') will amplified specific DNA fragment of $1300 \mathrm{bp}$. The PCR condition was carried out for 30 cycles including predenaturation step for $2 \mathrm{~min}$ at $94^{\circ} \mathrm{C}$, denaturation for 30 sec at $92^{\circ} \mathrm{C}$, annealing for $30 \mathrm{sec}$ at $55^{\circ} \mathrm{C}$, polymerization for $1 \mathrm{~min}$ at $75^{\circ} \mathrm{C}$ and post PCR for 10 min at $75^{\circ} \mathrm{C}$. The DNA fragments were purified and subsequently sequenced by P.T. Charoen Phokphan Indonesia (CPI). The sequence of each 16S rRNA gene of Pseudomonas spp. isolates were aligned for any similarity sequences on GenBank database by using the BLASTN program. The sequences were also aligned with other 16S rRNA gene from other Pseudomonas species with CLUSTALW program, in order to build a phylogenetic tree.

\section{RESULTS}

Isolation of Pseudomonas sp: A total 115 isolates Pseudomonas spp. was isolated from rhizosphere soil of soybean plants. All isolates were identified on the basis of morphological and physiological characters as described in Bergeys Manual of Determinative Bacteriology. Pseudomonas sp. are rod shaped 0.5$0.8 \times 1-3 \mu \mathrm{m}$, Gram negative, catalase positive and oxidase positive. There were 27 isolates $(24.3 \%)$ among 115 isolates which identified to have fluorescence activity (Table 1).

\section{Growth promoter assay:}

Measurement of IAA: 113 isolates of Pseudomonas spp. have detected to be able of producing IAA in various levels (Table 1), while 2 isolates showed no producing activity (Crb-7 and Crb-38). The biggest part of isolates $(67.8 \%)$ was detected of producing IAA in low level $(\leq 10 \mathrm{ppm})$. About $28.7 \%$ of isolates were capable to produce IAA in moderate level (11-19 ppm) and $17 \%$ isolates showed IAA producing activity in high level ( $\geq 20 \mathrm{ppm}$ ). The highest level of IAA was produced by Crb-90 (23, $04 \mathrm{ppm}$ ), while the lowest one was produced by $\mathrm{Crb}-100$ (0.33 ppm).

Phosphate solubilizing assay: 62 Pseudomonas sp isolates were able to solubilize tri-calcium phosphate in Pikovskaya medium (Table 1). The highest solubilizing index was performed by Pseudomonas sp Crb-2 (1.286), while the lowest one was performed by Pseudomonas sp Crb-66 (0.100). 
Am. J. Agri. \& Biol. Sci., 6 (1): 134-141, 2011

Table 1: Indole acetic acid and phosphate solubilizing production of Pseudomonas sp isolated from rhizosfer of soybean plant

\begin{tabular}{|c|c|c|c|c|c|c|c|c|}
\hline Isolates & IAA (ppm) & $\mathrm{SI}^{\mathrm{a}}$ & Isolates & IAA (ppm) & SI & Isolates & IAA (ppm) & SI \\
\hline Crb-100 & 0.333 & 0.400 & Crb-12 & 6.683 & 0.500 & Crb-66 & 10.677 & 0.1 \\
\hline Crb-99 & 0.810 & 0.279 & Crb-112 & 6.881 & - & Crb-111 & 10.929 & - \\
\hline Crb-94* & 1.127 & 0.238 & Crb-75 & 7.127 & - & Crb-59 & 11.268 & - \\
\hline Crb-92 & 1.444 & 0.417 & Crb-95* & 7.238 & 0.279 & Crb-106 & 11.524 & 0.600 \\
\hline Crb-91 & 1.524 & - & Crb-88 & 7.264 & - & Crb-96 & 11.603 & 0.613 \\
\hline Crb-27 & 1.978 & - & Crb-8* & 7.397 & - & Crb-58 & 11.661 & - \\
\hline Crb-25 & 2.198 & 0.040 & Crb-13 & 7.397 & 0.356 & Crb-72 & 11.740 & 0.250 \\
\hline Crb-3* & 2.317 & - & Crb-107 & 7.437 & - & Crb-71 & 11.898 & 0.297 \\
\hline Crb-37 & 2.343 & 0.625 & Crb-93* & 7.595 & 0.256 & Crb-28 & 12.161 & - \\
\hline Crb-32 & 2.599 & - & Crb-10 & 7.635 & - & Crb-61 & 12.291 & 0.365 \\
\hline Crb-29 & 2.635 & - & Crb-74* & 7.718 & 0.271 & Crb-26 & 12.526 & - \\
\hline Crb-34 & 2.818 & 0.500 & Crb-115 & 7.754 & - & Crb-35 & 12.526 & - \\
\hline Crb-33 & 3.255 & 0.875 & Crb-18 & 7.820 & 0.515 & Crb-5 & 12.754 & 0.500 \\
\hline Crb-19 & 3.367 & 0.867 & Crb-76 & 7.855 & - & Crb-23 & 13.172 & - \\
\hline Crb-14 & 3.445 & - & Crb-60* & 8.039 & - & Crb-97 & 13.548 & 0.467 \\
\hline Crb-22 & 3.445 & - & Crb-65 & 8.118 & 0.059 & Crb-15* & 13.992 & - \\
\hline Crb-41 & 3.730 & 0.400 & Crb-6 & 8.349 & 0.633 & Crb-21 & 14.148 & - \\
\hline Crb-44* & 3.730 & - & Crb-11 & 8.349 & - & Crb-69 & 14.417 & 0.270 \\
\hline Crb-39 & 3.912 & - & Crb-67 & 8.433 & 0.133 & Crb-70 & 14.457 & 0.338 \\
\hline Crb-30 & 4.314 & - & Crb-40 & 8.657 & 0.600 & Crb-84* & 14.627 & 0.556 \\
\hline Crb-36 & 4.387 & 0.503 & Crb-55 & 8.787 & 0.350 & Crb-24 & 15.164 & - \\
\hline Crb-101 & 4.387 & - & Crb-9 & 8.984 & - & Crb-46 & 15.795 & 0.589 \\
\hline Crb-80 & 4.445 & 0.306 & Crb-48 & 8.984 & 0.143 & Crb-17* & 16.023 & 0.057 \\
\hline Crb-42 & 4.606 & 0.412 & Crb-57 & 9.181 & 0.650 & Crb- 49 & 16.189 & 0.256 \\
\hline Crb-16* & 4.891 & 0.533 & Crb-64 & 9.181 & 0.143 & Crb-108 & 16.408 & - \\
\hline Crb-83 & 5.036 & - & Crb-103 & 9.183 & 0.667 & Crb-53 & 16.858 & 0.800 \\
\hline Crb-43 & 5.080 & - & Crb-109 & 9.183 & - & Crb-54 & 16.898 & - \\
\hline Crb-20 & 5.164 & - & Crb-113 & 9.302 & - & Crb-52 & 17.528 & 0.489 \\
\hline Crb-31 & 5.445 & - & Crb-4 & 9.460 & 0.976 & Crb-73 & 17.646 & 0.202 \\
\hline Crb-89 & 5.445 & 0.279 & Crb-2 & 9.579 & 1.286 & Crb-98 & 18.071 & 0.450 \\
\hline Crb-110 & 5.849 & - & Crb-114 & 9.619 & - & Crb-56 & 18.472 & 0.369 \\
\hline Crb-77 & 6.036 & - & Crb-45 & 9.654 & - & Crb-81 & 18.673 & - \\
\hline Crb-87 & 6.264 & - & Crb-105 & 9.738 & 0.750 & Crb-68 & 19.299 & 0.533 \\
\hline Crb-62 & 6.307 & 0.225 & Crb-50 & 10.008 & 0.500 & Crb-79 & 19.673 & - \\
\hline Crb-86 & 6.309 & 0.167 & Crb-51 & 10.165 & 0.530 & Crb-85 & 19.718 & 0.425 \\
\hline Crb-78 & 6.400 & - & Crb-1* & 10.214 & 0.208 & Crb-104 & 20.492 & 0.333 \\
\hline Crb-102 & 6.484 & 0.500 & Crb-47 & 10.362 & 0.388 & Crb-90 & 23.036 & - \\
\hline Crb-82* & 6.627 & - & Crb-63 & 10.598 & - & & & \\
\hline
\end{tabular}

Notes: Each number is mean of three replicates. Isolates with bold characters indicates group of fluorescence Pseudomonads; *: Isolates which

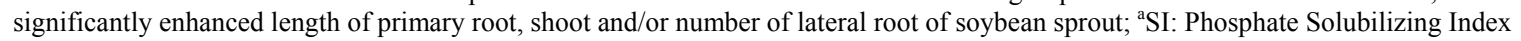

Germinating seed assay: Based on the bioassay, there were 17 isolates have determined to posses promoting seed growth activities. Nevertheless, there were only two isolates (Crb-44 and 63) which exhibited promoting activity for all parameters. Meanwhile, Crb$1,8,7,3,16$ and 93 were observed to be able of elongate shoot growth. Crb-35, 60, 82 and 84 were able to increase number of lateral root. Crb-15 showed promoting characters of increasing number of lateral root and length of shoot growth. Crb-94 increased the length of primary root and shoot. Moreover, Crb-17 and Crb-74 showed elongation activity of primary root and number of lateral root, respectively (Table 1).

Hypersensitivity test: Hypersensitivity test on tobacco leaves was conducted for each 17 isolates. Three isolates (Crb-7, Crb-35, Crb-63) exhibited positive hypersensitivity responses on tissue's leaves or indicated as pathogenic bacteria. The rest 14 isolates showed negative hypersensitivity responses. Thereby, we discarded 3 pathogenic isolates for subsequent assays.

\section{Biocontrol assays:}

Siderophore and chitinase production: We used the generic method to investigate siderophore's producing isolate qualitatively. It confirmed by the development of orange halos around bacterial colonies. By using CAS agar medium, over 14 isolates, there were 4 isolates (Crb-8, Crb-15, Crb-74 and Crb-84), 7 isolates (Crb-1, Crb-16, Crb-17, Crb-44, Crb-60, Crb-94 and Crb-95) and one isolates (Crb-3) were produced siderophore in low, moderate and high level, respectively (Table 2). Six isolates were observed to have chitinolytic activity in chitin agar medium (Table 2). The highest chitinolytic index was exhibited by Crb-3 (0.39). 
Am. J. Agri. \& Biol. Sci., 6 (1): 134-141, 2011

Table 2: Siderophre and Chitinase production of Pseudomonas sp isolates and heir antagonism against phytopathogenic fungi

\begin{tabular}{|c|c|c|c|c|c|}
\hline \multirow[b]{2}{*}{ Isolate } & \multirow[b]{2}{*}{ Siderophore $^{\mathrm{a}}$} & \multirow{2}{*}{$\begin{array}{l}\text { Chitinase } \\
\text { (CI) }\end{array}$} & \multicolumn{3}{|c|}{ Antagonism assays ${ }^{\mathrm{c}}$} \\
\hline & & & F. oxysporum & S. rolfsii & R.solani \\
\hline$\overline{\mathrm{Crb}-1}$ & ++ & 0.3 & - & - & - \\
\hline Crb-3 & +++ & 0.39 & - & - & +++ \\
\hline Crb- 8 & + & 0.32 & + & - & ++ \\
\hline Crb-15 & + & 0.32 & - & - & - \\
\hline Crb-16 & ++ & - & +++ & - & + \\
\hline Crb-17 & ++ & - & +++ & - & - \\
\hline Crb-44 & ++ & - & +++ & + & + \\
\hline Crb-60 & ++ & - & + & - & + \\
\hline Crb-74 & + & - & - & + & + \\
\hline Crb- 82 & - & - & + & - & +++ \\
\hline Crb- 84 & + & 0.29 & + & - & - \\
\hline Crb-93 & - & - & - & - & - \\
\hline Crb-94 & ++ & 0.32 & - & +++ & - \\
\hline Crb-95 & ++ & - & - & - & + \\
\hline
\end{tabular}

Notes: All the experiments were done in two replications; ${ }^{\text {a }}+$ : Low concentration; ++: Moderate concentration, $+++:$ High concentration, $-:$ No siderophore detected; ${ }^{b}$. -: No phosphate solubilization; ${ }^{c}$. +: Inhibition indeks $\leq 10 \%$; ++: Inhibition index $11-20 \%$; +++: Inhibition indeks $\geq 20 \%$; -: No inhibition activity detected

Table 3: Homology search analysis of 16S rRNA gene sequences analysis using BLASTN programs

\begin{tabular}{llll}
\hline Isolate code & Homolog with & Identity (\%) & Accession number \\
\hline Crb-1 & Pseudomonas sp. 12M16 & 91 & AB120346.1 \\
Crb-3 & Pseudomonas monteilii strain R23 & 95 & DQ095885.1 \\
Crb-8 & Pseudomonas sp. 12M16 & 91 & AB120346.1 \\
Crb-15 & Pseudomonas sp. 12M16 & 94 & AB120346.1 \\
Crb-16 & Pseudomonas sp. m41 & 94 & EU375659.1 \\
Crb-17 & Pseudomonas plecoglossicida strain NyZ12 & 99 & EF544606.2 \\
Crb-44 & Pseudomonas beteli & 99 & AB021406 \\
Crb-60 & Pseudomonas aeruginosa strain NBRAJG90 & EU734822.1 \\
Crb-74 & Pseudomonas putida strain HRB-4 & 92 & DQ870553.1 \\
Crb-82 & Pseudomonas sp. RW10S1 & 86 & AM911688.1 \\
Crb-84 & Pseudomonas sp. BFF-1 & 93 & EF031081.1 \\
Crb-93 & Pseudomonas putida strain 24 2R3 & 92 & AM086255.1 \\
Crb-94 & Pseudomonas mosselii isolate 164 & 86 & EU244761.1 \\
Crb-95 & Pseudomonas sp. isolate R-20954 & 95 & AM114531 \\
\hline
\end{tabular}

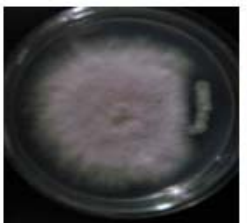

(A)

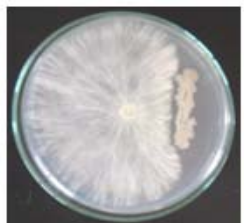

(B)

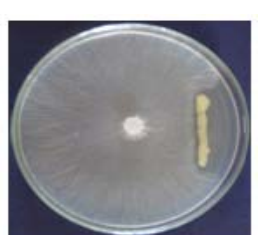

(C)
Fig. 1: (A) Antagonistic interaction between Pseudomonas sp. Crb-17 and F. oxysporum; (B) antagonistic interaction between Pseudomonas sp. Crb 94 and S. rolfsii; (C) antagonistic interaction between Pseudomonas sp. Crb 82 and $R$. solani on Potatose Dextrose Agar medium after 3-5 days incubation at room temperature

Antagonism assay against phytopathogenic fungi: Crb-16, Crb-17 and Crb-44 strongly inhibited the growth of $F$. oxysporum, whereas Crb-8, Crb-82 and Crb-84 were also able to inhibit $F$. oxysporum, even in low level. Only Crb-94 was detected to inhibit $S$. rolfsii in high level and two other isolates (Crb-44 and Crb74) weakly inhibited the growth of S. rolfsii. Instead of having inhibition activity against $F$. oxysporum, $\mathrm{Crb}-8$, Crb-16, Crb-44, Crb-60 and Crb-82 were also detected of having antagonism interaction to $R$. solani. Crb-44 was the only isolate that capable to inhibit all tested phytopathogens (Table 2 and Fig. 1).

Interesting facts in relation with biocontrol assay were exhibited by several isolates that possessed siderophore and chitinase activity but showed no inhibition activities against any phytopathogens tested (isolates Crb-1 and Crb-15). Opposite occurrence was revealed by Crb-82 which produced no active compounds (siderophore and chitinase), however, showed inhibiting activities against $F$. oxysporum and $R$. solani.

16S-rRNA gene amplification and phylogenetic tree construction: Partial $16 \mathrm{~S}$ r-RNA sequence analysis was conducted for all isolates that potentially promote germinating seeds and negative hypersensitivity responses on tobacco leaves. PCR amplification of $16 \mathrm{~S}$ rRNA gene yielded single $b$ and at approximately 1300 base pair (bp). All those 14 isolates were characterized as group of Pseudomonas genera and very diverse in species taxa (Table 3 ). 
Am. J. Agri. \& Biol. Sci., 6 (1): 134-141, 2011

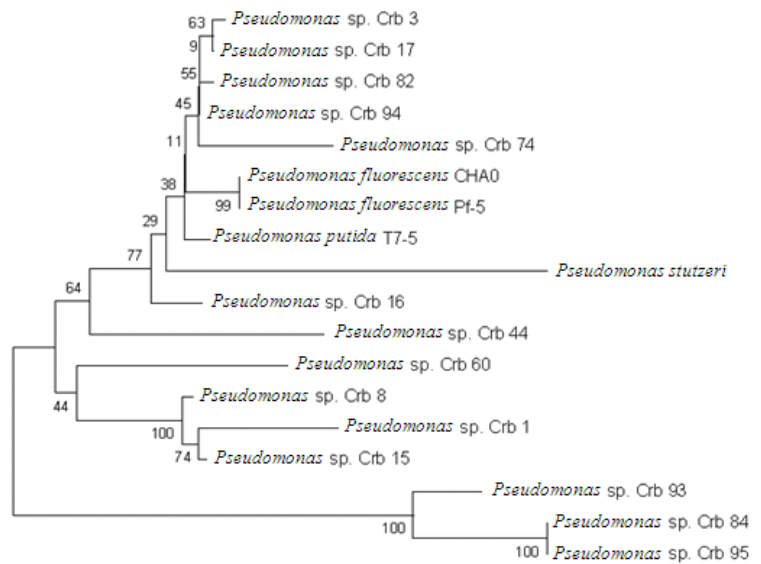

Fig. 2: Pylogenetic Tree of Pseudomonas sp. isolates based on 16S-rRNA gene sequence. Crb-1, Crb$15, \mathrm{Crb}-8$ and $\mathrm{Crb}-60$ were gathered into one group which all isolates detected to have excellent growth promoter activity. Other isolates (Crb-3, Crb-17, Crb-82, Crb-94 and Crb74) were grouped separately and closely related to Pf-5 and CHA0, these groups were detected as excellent biocontrol agent

As comparison for phylogenetic tree construction, we used 16S-rRNA sequence of $P$. fluorescens Pf-5 and $P$. fluorescens $\mathrm{CHA} 0$ as representatives for biocontrol Pseudomonads, meanwhile P. putida and P. stutzeri were used as representatives for growth promoter Pseudomonads.

According to the phylogenetic tree (Fig. 2), isolates Crb-3, Crb-17, Crb-82, Crb-94 and Crb-74 were grouped into group 1 which was highly related to the bicontrol agent $P$. fluorescens Pf-5 and $P$. fluorescens CHA0. Meanwhile, some isolates (Crb-60, Crb-15, Crb-8 and Crb-1) and isolates Crb-93, Crb-94 and Crb95 were grouped separately.

\section{DISCUSSION}

Certain strains of rhizosphere colonizing Pseudomonas sp. have gained world wide attention in recent years due to their abilities in promoting plant growth. Therefore, in this study, we further explore the existence of any indigenously Pseudomonas sp. isolated from 1 and of Indonesia that may broadly used at crop field as potential inoculants. The isolation of Pseudomonas sp. from rhizosphere soil was yielded 115 isolates with various morphological appearances. All those isolates were subsequently analyzed for plant growth promoting attributes.
The capability to increase plant growth parameters in germinating seed bioassay is highly related to the IAA level, which produced by Pseudomonas spp. isolates. Varying results of germinating seed assay had also pointed out that there was complex interaction between bacterial IAA and seedlings, thereby caused different responses of plant growth tendencies, yet there is stimulation of bacterial IAA to the development of the host plant root system (Patten and Glick, 2002). In addition, Patten and Glick (2002) had also investigated that, low levels of IAA can stimulate root elongation, while high levels of bacterial IAA, whether from IAAoverproducing mutants or strains that naturally secrete high levels or from high-density inoculua, stimulate the formation of lateral and adventitious roots. In this study there were various levels of IAA concentration that capable to promote seed growth. Thereby, it was difficult to conclude the optimum IAA concentration that may promote seed growth.

Instead of IAA it self, the main factor of promoting plant growth by bacterial IAA is root colonization. Bacterial producing IAA must survive in the environment make use of nutrients exuded by the plant root, proliferate and be able to efficiently colonize the entire root system (Bloemberg and Lugtenberg, 2001). So, it would be no beneficial effects for those isolates which showed high IAA producing activity but lack of root colonizing activity, as exhibited by 12 isolates which produced high level IAA $(\geq 16.5 \mathrm{ppm})$. Other complexes interactions between plant and bacterial producing IAA might be due to the fact that IAA secreted by a bacterium may promote root growth directly by stimulating plant cell elongation or cell division, or indirectly by influencing bacterial ACC deaminase activity. ACC deaminase produced by many plant growth-promoting bacteria including Pseudomonas genera, is involved in the stimulation of root elongation in seedlings. ACC deaminase hydrolyzes plant ACC, the intermediate precursor of the phytohormone ethylene and thereby prevents the production of plant growth-inhibiting levels of ethylene (Patten and Glick, 2002).

The ability of several isolates to solubilize tricalcium phosphate in vitro suggested the application of those isolates in crop fields. Rodriguez and Fraga (1999) had studied that Pseudomonas and other Phosphate Solubilizing Bacteria (PSB) like Bacillus and Rhizobium are capable to increase the availability of phosphor in soil. Specifically, all those isolates might be potential inoculants for alkaline soil based on the ability to solubilize phosphate bounded by calcium which mostly exists in alkaline soils, whereas in the acidic soil, $\mathrm{P}$ were mostly fixed by $\mathrm{Fe}$ or $\mathrm{Al}$ (Goldstein, 1995). 
Siderophore is one of the biocontrol mechanisms belonging to PGPR groups, including Pseudomonas sp. under iron limiting condition, PGPR produce a range of siderophore which have a very high affinity for iron. Therefore, the low availability of iron in the environment would suppress the growth of pathogenic organisms including plant pathogenic fungi (Whipps, 2001). Instead of siderophore, there are other mechanisms of biocontrolling. Haas and Defago (2005) had reviewed some biocontrolling mechanisms of Fluorescent Pseudomonads, include antibiotics compounds, eliciting Induced Systemic Resistance (ISR) of plant and lytic enzyme secretion. Here, we have observed six isolates that is chitinase positive.

Based on siderophore and chitinase production assay, we assumed that some isolates which produced those bioactive compounds are able to inhibit the phytopathogenic fungi, as shown by Crb-3. Nonetheless, there was no evidence to proclaim the exact biocontrol mechanisms. It is due to lots of biocontrol activities that may involved in controlling pathogens. Interestingly, some isolates like Crb-82 displayed no siderophore and chitinase producing activity, however there were inhibition activity against F. oxysporum and S. rolfsii. Therefore, we consider of other biocontrol activities that possessed by those isolates such as $\mathrm{HCN}$ or antibiotics that may entangled in inhibition activity.

The other fact was showed by Crb-1 and Crb-15 which were able to produce siderophore or chitinase, but lack of biocontrol activity. We consider of any specificity interaction between the bacteria or the bacterial biocompounds with phytopathogens, as described by Barea et al. (1998). The used of PDA on opposite assay against phytopathogens would also become a limiting factor for such biocompound like siderophore. Mostly, PGPR produces a range of siderophore under iron limiting condition (Whipps, 2001). Therefore, we assumed that PDA was not an appropriate medium for some isolates to produce siderophore due to the rich mineral availability in this medium.

16S-rRNA sequence analysis showed that all isolates were pretty diverse in species taxa. Interestingly, some isolates which showed good biocontrol activity (Crb-3, Crb-17, Crb-82, Crb-94 and Crb-74) were closely related to $P$. fluorescens $\mathrm{P}-5$ and P. fluorescens CHA0. Whereas, Crb-60, Crb-8, Crb-1 and Crb-15 which were observed to have excellent growth promoter activitiy were gathered in to one group.

To be based on splendid growth promoter and biocontrol activities, we proposed five isolates (Crb-3,
Crb-16, Crb-17, Crb-44 and Crb-94) as potential inoculants depending on each plant promoting attributes. Yet, we still have to analysis any appropriate assays that may support these isolates to be applicable in crop fields.

\section{CONCLUSION}

Five strains of Pseudomonas sp. were identified as potential plant growth promoting rhizobacteria, including Crb-3, Crb-16, Crb-17, Crb-44 and Crb-94. Most of these isolates are belonging to the group of fluorescens pseudomonads. We consider for further in planta research to determine their activities in promoting plant growth.

\section{ACKNOLEDGMENT}

This research was funded by Incentive Program for Basic Research ("Program Insentif Riset Dasar") from the Ministry Department of Research and Technology of Indonesia (KNRT) 2007-2009 to ATW. Therefore, we thank and appreciate for all the supports given to us, in order to do this research.

\section{REFERENCES}

Ahmad, F., I. Ahmad and M.S. Khan, 2005. Indole acetic acid production by the indigenous e of Azotobacter and fluorescent Pseudomonas in the presence and absence of tryptophan. Turk. J. Biol., 29: 29-34.

Bai, Y., X. Zhou and D.L. Smith, 2003. Enhanced soybean plant growth resulting from coinoculation of Bacillus strains with Bradyrhizobium japonicum. Crop Sci., 43: 1774-1781. DOI: 10.2135/CROPSCI2003.1774

Bano, N. and J. Musarrat, 2003. Isolation and characterization of phorate degrading soil bacteria of environmental and agronomic. Lett. Applied Microbiol., 36: 349-353. DOI: 10.1046/J.1472765x.2003.01329.X

Barea, J.M., G. Andrade, V.V. Bianciotto, D. Dowling and S. Lohrke et al., 1998. Impact on arbuscular mycorrhiza formation of pseudomonas strains used as inoculants for biocontrol of soil-borne fungal plant pathogens. Applied Environ. Microbiol., 64: 2304-2307.

Bloemberg, G.V. and B.J.J Lugtenberg, 2001. Molecular basis of plant growth promotion and biocontrol by rhizobacteria. Curr. Opinion. Plant. Biol., 4: 343-350. DOI: 10.1016/S13695266(00)00183-7 
Cattelan, A.J., P.G. Hartel and J.J. Fuhrmann, 1999. Screening for plant growth-promoting rhizobacteria to promote early soybean growth. Soil. Sci. Soc. Am. J., 63: 1670-1680. DOI: 10.2136/Sssaj1999.6361670x

Dey, R., K.K. Pal, D.M. Bhatt and S.M. Chauhan, 2004. Growth promotion and yield enhancement of peanut (Arachis hypogaea L.) by application of plant growth-promoting rhizobacteria. Microbiol. Res., 159: 371-394. DOI: 10.1016/J.MICRES.2004.08.004

Dikin, A., K.Sijiam, J. Kadir and I.A. Seman, 2006. Antagosnistic bacteria against Schizophyllum commune fr. in Peninsular Malaysia. Biotropia, 13: 111-121.

Goldstein, A.H., 1995. Recent progress in underst anding the molecular genetics and biochemistry of calcium phosphate sulubilization by gram negative bacteria. Biol. Agric. Hort., 12: 185-193.

Haas, D. and G. Defago, 2005. Biological control of soil-borne pathogens by fluorescent pseudomonads. Nature. Rev. Microbiol., 3: 307-319. DOI: $10.1038 /$ nrmicro1129

Husen, E., 2003. Screening of soil bacteria for plant growth promotion activities in vitro. Indones. J. Agric. Sci., 4: 27-31.

Lelliott, R.A. and D.E. Stead, 1987. Methods for the Diagnosis of Bacterial Diseases of Plants. 1st Edn., Blackwell Scientific Publications, London, ISBN10: 0632012331, pp: 216.

Marchesi, J.R., T. Sato, A.J. Weightman, T.A. Martin and J.C. Fry et al., 1998. Design and evaluation of useful bacterium-specific PCR primers that amplify genes coding for bacterial 16S-rRNA. Applied Environ. Microbiol., 64: 795-799.
Mubarik, N.R., I. Mahagiani, A. Anindyaputri, S. Santoso and I. Rusmana, 2010. Chitinolytic bacteria isolated from chili rhizosphere: Chitinase characterization and its application as biocontrol for whitefly (Bemisia tabaci Genn.). Am. J. Agric. Biol. Sci., 5: 430-435. DOI: 10.3844/ajabssp.2010.430.435

Patten, C.L. and B.R. Glick, 2002. Role of pseudomonas putida indoleacetic acid in development of the host plant root system. Applied Environ. Microbiol., 68: 3795-3801. DOI: 10.1128/AEM.68.8.3795-3801.2002

Ramesh, K.N., A.V. Thirumalai and P. Gunasekaran, 2002. Genotyping of antifungal compounds producing plant growth-promoting rhizobacteria, Pseudomonas fluorescens. Curr. Sci., 82: 1463-1466.

Rao, S.W.C.B. and M.K. Sinha, 1962. Phosphate dissolving microorganism in the soil and rhizosphere. Ind. J. Sci., 23: 272-278.

Rodriguez, H. and R. Fraga, 1999. Phosphate solubilizing bacteria and their role in plant growth promotion. Biotechnol. Adv., 17: 319-339. DOI: 10.1016/S0734-9750(99)00014-2

Vasanthakumar, A. and P.S. McManus, 2004. Indole-3acetic acid-producing bacteria are associated with cranberry stem gall. Phytopathology, 94: 1164-1171. DOI: 10.1094/PHYTO.2004.94.11.1164

Whipps, J.M., 2001. Microbial interactions and biocontrol in the rhizosphere. J. Exp. Bot., 52: 487511. DOI: $10.1093 /$ jexbot/52.suppl 1.487

Wilson, K., 1994. Preparation of Genomic DNA from Bacteria. Curr. Protoc. Mol. Biol., PMID: 18265184

Wu, S.C., Z.H. Cao, Z.G. Li, K.C. Cheung and M.H. Wonga, 2005. Effects of biofertilizer containing Nfixer, $\mathrm{P}$ and $\mathrm{K}$ solubilizers and AM fungi on maize growth: A greenhouse trial. Geoderma, 125: 155-166. DOI: 10.1016/J.GEODERMA.2004.07.003 